\title{
PRINCIPAL REES QUOTIENTS OF FREE INVERSE SEMIGROUPS
}

\author{
D. EASDOWN \\ School of Mathematics and Statistics, University of Sydney, Sydney, NSW 2006, Australia \\ e-mail:de@maths.usyd.edu.au \\ and L. M. SHNEERSON \\ Department of Mathematics and Statistics, Hunter College, The City University of New York, \\ 695 Park Avenue, New York, NY 10021, U.S.A. \\ e-mail:lms6@nyu.edu
}

(Received 25 January, 2002; accepted 2 August, 2002)

\begin{abstract}
We prove that up to isomorphism $\langle a, b \mid a b=0\rangle$ is the unique principal Rees quotient of a free inverse semigroup that is not trivial or monogenic with zero, satisfying a nontrivial identity in signature with involution.
\end{abstract}

2000 Mathematics Subject Classification. 20M05, 20M18, 20 M99.

1. Introduction. In [9] the authors initiated the study of identities satisfied by finitely presented Rees quotients of free inverse semigroups. It was proved [9, Section 5] that a semigroup from that class satisfies a nontrivial semigroup identity if and only if its growth is polynomial. Key ingredients were a graphical technique due to Ufnarovsky [10], [11], Adjan's identity for the bicyclic semigroup [1], and the fact (easily deduced from [8]) that the bicyclic and free monogenic inverse semigroups satisfy the same semigroup identities. By contrast, however, an example was given in [9, Section 4] of a semigroup from that class that has exponential growth yet satisfies a nontrivial identity in signature with involution. In this paper we examine principal Rees quotients of free inverse semigroups; that is, Rees quotients by a principal ideal. We show that up to isomorphism

$$
S=\langle a, b \mid a b=0\rangle
$$

(regarded as a presentation as an inverse semigroup with zero) is the unique member of this class that is not trivial or monogenic with zero yet satisfies a nontrivial identity in signature with involution. Of course $S$ has exponential growth (since $a$ and $b^{-1}$ generate a noncyclic free subsemigroup), but $S$ does not contain any nonmonogenic free inverse subsemigroups.

2. Preliminaries. We assume familiarity with the basic definitions and elementary results from the theory of inverse semigroups that can be found in any of [2], [3], [4] or [6]. We denote the free semigroup, free inverse semigroup and free group over an alphabet $A$ by $F_{A}, F I_{A}$ and $G_{A}$, respectively. Equality in free semigroups will be denoted by $\underline{\overline{ }}$. Recall that a word $w$ is reduced if $w$ does not contain $x x^{-1}$ as a subword for any letter $x \in A \cup A^{-1}$. If $w \in F_{A \cup A^{-1}}$ then we denote by $\bar{w}$ the reduced 
word equivalent to $w$ in $G_{A}$. Without causing confusion we shall identify elements of $F I_{A}$ and $G_{A}$ with words over the alphabet $A \cup A^{-1}$. Then the mapping $w \mapsto \bar{w}$ induces a homomorphism from $F I_{A}$ to $G_{A}$. Denote by $[u, v]$ the commutator $u v u^{-1} v^{-1}$.

Recall that elements of $F I_{A}$ may be regarded as birooted word trees (introduced in [5]), the terminology and theory of which are explained in [3]. (See also [9, Section 2].) If $u$ and $v$ are elements of $F I_{A}$, then the word tree of $u$ is a subtree of the word tree of $v$ if and only if $v$ may be expressed as a product of elements, one of whose factors is $u$, in which case we say that $u$ divides $v$. Any reference to Green's relation $\mathcal{J}$ will be with respect to $F I_{A}$. Note that two words over $A \cup A^{-1}$ are $\mathcal{J}$-related if and only if their word trees are identical.

The following lemma can be verified easily using Reilly's criterion [7, Theorem 2.2].

LEMMA 2.1. If $\epsilon, \delta, v, \eta \in\{ \pm 1\}$ and $\epsilon \neq v$ or $\delta \neq \eta$ then $a^{\epsilon} b^{\delta}$ and $a^{v} b^{\eta}$ freely generate an inverse subsemigroup of $F I_{\{a, b\}}$.

Denote by $\langle u, v\rangle$ the inverse subsemigroup of $F I_{A}$ generated by elements $u$ and $v$. The next lemma follows easily by inspection of word trees.

LEMMA 2.2. Suppose that $a$ and $b$ are letters from $A$, that ab divides a word $c$ in $F I_{A}$ and that $c$ divides elements from each of $\left\langle a b^{-1}, a^{-1} b\right\rangle,\left\langle a b, a b^{-1}\right\rangle$ and $\left\langle a^{-1} b, a b\right\rangle$. Then $c \mathcal{J} a b$.

An identity in signature with involution is an equation of the form

$$
P=Q
$$

where $P$ and $Q$ are words (also called terms) from the free semigroup $F_{V \cup V^{-1}}$, where $V=$ $\left\{x_{1}, \ldots, x_{n}\right\}$ is some finite alphabet (of variables). We say that an inverse semigroup $S$ satisfies this identity if the equality (1) holds in $S$ when each side is evaluated after substituting arbitrary elements of $S$ for $x_{1}, \ldots, x_{n}$. We call the identity (1) trivial if it is satisfied by all inverse semigroups, nontrivial otherwise. By [7, Corollary 2.7] the free inverse semigroup $\mathrm{FI}_{2}$ on two generators contains a copy of the free inverse semigroup on countably infinitely many generators, so that any identity satisfied by $F I_{2}$ must be satisfied by all inverse semigroups. Thus if $F I_{2}$ embeds in an inverse semigroup $S$, then $S$ cannot satisfy any nontrivial identity in signature with involution.

3. Principal Rees quotients. In this section we prove a sequence of lemmas culminating in a theorem which gives an identity in signature with involution satisfied by the semigroup $S=\langle a, b \mid a b=0\rangle$. We then prove that $S$ is unique amongst the class of nontrivial nonmonogenic principal Rees quotients of free inverse semigroups that satisfy any such identity.

Throughout, denote by $X$ the subsemigroup of $S$ generated by $a$ and $b^{-1}$. It is clear that multiplication in $S$ of words from $X$ is simply concatenation, so that $a$ and $b^{-1}$ are free generators of $X$.

LEMMA 3.1. Let $w$ be a nonempty reduced word that is nonzero in $S$. Then $w \underline{\overline{ }} w_{1}^{-1} w_{2}$ for some $w_{1}, w_{2} \in X^{1}$.

Proof. Observe that $a b=b^{-1} a^{-1}=0$ in $S$. Let $u$ be any reduced word that is nonzero in $S$. (i) If $u$ begins with $a$ or $b^{-1}$, then $u \in X$. (ii) If $u$ ends with $a^{-1}$ or $b$, then $u^{-1} \in X$. Thus we may suppose that $w$ does not begin with $a$ or $b^{-1}$ and does not end with $a^{-1}$ or $b$, for otherwise the conclusion of the lemma holds trivially. If $w$ does not 
contain $b^{-1}$, then $w \overline{\mathrm{o}} v a^{\theta}$, for some positive integer $\theta$ and some word $v$ ending with $b$, so that $a^{\theta}, v^{-1} \in X$ and the claim of the lemma follows. Suppose then that $w$ contains $b^{-1}$, so that $w \underline{\mathrm{o}} u v$, for some $u$ not containing $b^{-1}$ and $v$ beginning with $b^{-1}$. Then $v \in X$. Also $u$ does not end with $b$ since $w$ is reduced. Either $u$ ends with $a^{-1}$, in which case $u^{-1} \in X$ and we are done, or $u$ ends with $a$. In the latter case $u \underline{\overline{0}} u_{0} a^{\theta}$, for some $\theta>0$ and $u_{0}$ ending with $b$, whence $u_{0}^{-1}, a^{\theta} v \in X$ and again the claim of the lemma follows, completing the proof.

LemMA 3.2. Let $u, v$ be nonempty reduced words such that $u, v \in X$ and $u v^{-1} \neq 0$ in $S$. Then either $u$ is a suffix of $v$ or $v$ is a suffix of $u$. In particular, if $u$ and $v$ have the same length, then $u \underline{\bar{\Xi}} v$.

Proof. If $u$ and $v$ end with different letters, then without loss of generality $u \underline{\overline{\mathrm{O}}} u_{0} a, v \underline{\overline{\mathrm{O}}} v_{0} b^{-1}$, for some $u_{0}, v_{0}$, so that $u v^{-1} \underline{\mathrm{\sigma}} u_{0} a b v_{0}=0$ in $S$, a contradiction. Hence $u$ and $v$ end with the same letter so that there is a nonempty maximal suffix $x \in X$ such that $u \underline{\overline{ }} u_{0} x, v \underline{\bar{\sigma}} v_{0} x$, for some $u_{0} v_{0}$ in $X^{1}$. Suppose that $u_{0}$ and $v_{0}$ are both nonempty. Then $u_{0} v_{0}^{-1} \neq 0$ in $S$, because $u_{0} v_{0}^{-1}$ divides $u v^{-1}$. Hence, from above, $u_{0}$ and $v_{0}$ end with the same letter, contradicting the maximality of $x$. Hence either $u_{0}$ or $v_{0}$ is empty, and the lemma is proved.

LEMMA 3.3. Let $u$ and $v$ be nonempty reduced words in $X$ such that the commutator

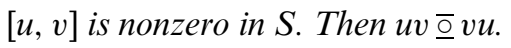

Proof. Observe that $u v$ and $v u$ are nonempty reduced words of the same length lying in $X$, and $(u v)(v u)^{-1} \neq 0$ in $S$. Hence $u v \underline{\underline{a}} v u$ by Lemma 3.2.

Lemma 3.4. Let $w$ be a reduced word such that $w^{2} \neq 0$ in $S$. Then $w \underline{\underline{ }} w_{1}^{-1} w_{2} w_{1}$ or $w \overline{\mathrm{D}} w_{1}^{-1} w_{2}^{-1} w_{1}$, for some $w_{1}, w_{2} \in X$.

Proof. By Lemma 3.1, $w \underline{\bar{\Xi}} u^{-1} v$, for some $u, v \in X$. Then $v u^{-1}$ is a divisor of $w^{2}$ and so is nonzero in $S$. By Lemma 3.2, either $u$ is a suffix of $v$ or $v$ is a suffix of $u$, and the conclusion of the lemma follows.

LEMMA 3.5. Suppose that $u \in X, v \underline{\bar{\Xi}} x_{1}^{-1} x_{2} x_{1}$, for some $x_{1}, x_{2} \in X$, and $v u^{2} v u^{-1} v^{-1}$ is nonzero in $S$. Then $\overline{u v}$ 을.

Proof. Certainly $u x_{1}^{-1}$ is nonzero in $S$ since it divides $v u^{2} v u^{-1} v^{-1}$. Hence, by Lemma 3.2, either $u$ is a suffix of $x_{1}$ or $x_{1}$ is a suffix of $u$. Suppose first that $x_{1} \overline{\mathrm{o}} y u$, for some $y \in X$. Certainly $y u y^{-1}$ is nonzero in $S$ since it also divides $v u^{2} v u^{-1} v^{-1}$. Hence, by Lemma 3.2 again, $y$ is a suffix of $y u$ and so $\overline{y u y^{-1}} \in X$. Further $v u^{2} v u^{-1} v^{-1}$ is divided by $y u^{2} y^{-1} x_{2} y u^{-1} y^{-1} x_{2}^{-1}$, which in turn is divided by $y u y^{-1} x_{2} y u^{-1} y^{-1} x_{2}^{-1}$, since $y$ is a suffix of $y u$. Hence $\left[\overline{y u y^{-1}}, x_{2}\right]$ is nonzero in $S$, so that $\overline{y u y^{-1}} x_{2} \overline{\bar{O}} x_{2} \overline{y u y^{-1}}$, by Lemma 3.3. It follows that $\overline{u v} \underline{\underline{ }} \overline{v u}$. Suppose now that $u \underline{\underline{\alpha}} u_{0} x_{1}$, for some $u_{0} \in X$. Then $u^{2} v u^{-1} v^{-1}$ is divided by $\left[x_{1} u_{0}, x_{2}\right]$, so that the latter is nonzero in $S$. Hence $x_{1} u_{0} x_{2} \overline{\mathrm{o}} x_{2} x_{1} u_{0}$, by Lemma 3.3. It follows again that $\overline{u v} \underline{\underline{\mathrm{o}}} \bar{v}$, and the lemma is proved.

LEMMA 3.6. Let $u, v \in S$ and suppose that each of

$$
v u^{2} v\left(u v^{2} u\right)^{-1}, \quad\left(v u^{2} v\right)^{-1} u v^{2} u, \quad v^{-1} u^{2} v^{-1}\left(u v^{-2} u\right)^{-1}, \quad\left(v^{-1} u^{2} v^{-1}\right)^{-1} u v^{-2} u
$$

is nonzero in $S$. Then $\overline{u v} \underline{\underline{ }} \overline{v u}$.

Proof. Without loss of generality we may suppose that $u$ and $v$ are reduced. Certainly $u^{2}$ and $v^{2}$ are nonzero in $S$ so that, by Lemma 3.4, either $u$ or $u^{-1}$ 으 $w_{1}^{-1} w_{2} w_{1}$ 
and either $v$ or $v^{-1} \underline{\overline{0}} w_{3}^{-1} w_{4} w_{3}$, for some $w_{1}, w_{2}, w_{3}, w_{4} \in X$. The four expressions listed in the hypothesis of the lemma are permuted by interchanging $u$ with $u^{-1}$ or $v$ with $v^{-1}$. Hence, without loss of generality we may suppose that $u \underline{\overline{0}} w_{1}^{-1} w_{2} w_{1}$ and $v \underline{\overline{0}} w_{3}^{-1} w_{4} w_{3}$. Certainly $u v \neq 0$ in $S$, and so $w_{1} w_{3}^{-1}$, being a divisor of $u v$, is nonzero in $S$. Hence, by Lemma 3.2, $w_{1}$ is a suffix of $w_{3}$ or $w_{3}$ is a suffix of $w_{1}$. Suppose that $w_{3} \underline{\overline{ }} w_{5} w_{1}$, for some $w_{5} \in X$. Put $u_{0} \underline{\mathrm{\Xi}} w_{2}$ and $v_{0} \underline{\mathrm{\Xi}} w_{5}^{-1} w_{4} w_{5}$. Then $v_{0} u_{0}^{2} v_{0} u_{0}^{-1} v_{0}^{-1} \neq 0$ in $S$ since it divides $v u^{2} v\left(u v^{2} u\right)^{-1}$. Hence, by Lemma 3.5, $\overline{u_{0} v_{0}} \underline{\underline{ }} \overline{v_{0} u_{0}}$, so that

$$
\overline{u v} \underline{\overline{\mathrm{o}}} \overline{w_{1}^{-1} u_{0} v_{0} w_{1}} \overline{\mathrm{o}} \overline{w_{1}^{-1} v_{0} u_{0} w_{1}} \overline{ } \overline{v u}
$$

and we are done. The case in which $w_{3}$ is a suffix of $w_{1}$ follows from the previous case because $v u^{2} v\left(u v^{2} u\right)^{-1}$ is inverted by interchanging $u$ and $v$, and the proof is complete.

THEOREM 3.7. $S=\langle a, b \mid a b=0\rangle$ satisfies the identity

$$
P Q=Q P
$$

where

$$
P=P(x, y)=\left[y x^{2} y, x^{-1} y^{-2} x^{-1}\right]
$$

and

$$
Q=Q(x, y)=\left[y^{-1} x^{2} y^{-1}, x^{-1} y^{2} x^{-1}\right] .
$$

Proof. Let $u, v \in S$. If $P(u, v)$ or $Q(u, v)$ is zero in $S$ then (2) holds trivially when $u, v$ are substituted for $x, y$ respectively. Suppose that $P(u, v)$ and $Q(u, v)$ are nonzero in $S$. In particular

$$
v u^{2} v\left(u v^{2} u\right)^{-1}, \quad\left(v u^{2} v\right)^{-1} u v^{2} u, \quad v^{-1} u^{2} v^{-1}\left(u v^{-2} u\right)^{-1}, \quad\left(v^{-1} u^{2} v^{-1}\right)^{-1} u v^{-2} u
$$

are all nonzero in $S$, so that $\overline{u v} \underline{\overline{0}} \overline{v u}$, by Lemma 3.6. Hence $\overline{P(u, v)}$ and $\overline{Q(u, v)}$ are empty so that $P(u, v)$ and $Q(u, v)$ are idempotents and thus commute. Hence (2) holds when $u, v$ are substituted for $x, y$ respectively, and the theorem is proved.

THEOREM 3.8. Let $T=\langle A \mid c=0\rangle$, for some word c over $A \cup A^{-1}$. Then $T$ satisfies a nontrivial identity in signature with involution if and only if $T$ is trivial, monogenic with zero or isomorphic to $\langle a, b \mid a b=0\rangle$.

Proof. The 'if part' is immediate by Theorem 3.7. Suppose that $T$ satisfies a nontrivial identity in signature with involution. In particular $T$ contains no nonmonogenic free inverse subsemigroup. Suppose $A$ contains at least 3 distinct letters $a_{1}, a_{2}, a_{3}$. Then each of $\left\langle a_{1}, a_{2}\right\rangle,\left\langle a_{1}, a_{3}\right\rangle$ and $\left\langle a_{2}, a_{3}\right\rangle$ is a nonmonogenic free inverse subsemigroup of $F I_{A}$ so that each contains an element divided by $c$. But by inspection this is impossible. Hence $A$ has at most 2 letters. If $A$ has 1 letter then $S$ is trivial or monogenic with zero. Suppose finally that $A=\{a, b\}$. If $c$ is $\mathcal{J}$-equivalent to a single letter, then $S$ is monogenic with zero. Suppose that $c$ is not $\mathcal{J}$-equivalent to a single letter. Without loss of generality we may suppose that $a^{2}$ or $a b$ divides $c$. By Lemma 2.1, the inverse subsemigroups $\left\langle a b^{-1}, a^{-1} b\right\rangle,\left\langle a b, a b^{-1}\right\rangle$ and $\left\langle a^{-1} b, a b\right\rangle$ are free of rank 2, so that $c$ must divide elements from each of them. It is impossible however that $a^{2}$ divides an element of $\left\langle a b, a b^{-1}\right\rangle$. Hence $a b$ divides $c$, and so, by Lemma 2.2, $c \mathcal{J} a b$, and $T$ is isomorphic to $\langle a, b \mid a b=0\rangle$, completing the proof. 
ACKNOWLEDGEMENT. The authors gratefully acknowledge the support of the Australian Research Council and the School of Mathematics and Statistics at the University of Sydney, which made possible this collaborative work.

\section{REFERENCES}

1. S. I. Adjan, Identities of special semigroups, Dokl. Acad. Nauk. 143, No. 3 (1962), 499-502 (in Russian).

2. A. H. Clifford and G. B. Preston, The algebraic theory of semigroups, Math. Surveys, Vol. I and Vol. II (Amer. Math. Soc., Providence, Rhode Island, 1961 and 1967).

3. P. M. Higgins, Techniques of semigroup theory (Oxford University Press, 1992).

4. J. M. Howie, An introduction to semigroup theory (Academic Press, 1976).

5. W. D. Munn, Free inverse semigroups, Proc. Lond. Math. Soc. (3) 29 (1974), 385-404.

6. M. Petrich, Inverse semigroups (Wiley \& Sons, 1984).

7. N. R. Reilly, Free generators of free inverse semigroups, Bull. Austral. Math. Soc. 7 (1972), 407-24; Corrigenda, ibid. 9 (1973), 479.

8. H. E. Scheiblich, A characterization of a free elementary inverse semigroup, Semigroup Forum 2 (1971), 76-79.

9. L. M. Shneerson and D. Easdown, Growth and satisfiability of identities in a class of finitely presented inverse semigroups with zero, Internat. J. Algebra Comput. 6 (1996), 105-121.

10. V. A. Ufnarovsky, Growth criterion for graphs and algebras given by words, Mat. Zam. 31, No. 3 (1982), 465-472 (in Russian).

11. V. A. Ufnarovsky, Combinatorial and asymptotic methods in algebra, VINITI Modern Problems of Mathematics, Fundamental Directions, Volume 57 (Moscow, 1990) (in Russian). 